\title{
Spin-polarized states of nuclear matter
}

\author{
W. Zuo ${ }^{1,2}$, U. Lombardo ${ }^{2,3}$ and C. W. Shen ${ }^{2}$ \\ 1 Institute of Modern Physics, Lanzhou, China \\ ${ }^{2}$ INFN-LNS, 44 Via S. Sofia, I-95123 Catania, Italy \\ 3 Dipartimento di Fisica, 57 Corso Italia, I-95129 Catania, Italy
}

\begin{abstract}
The equations of state of spin-polarized nuclear matter and pure neutron matter are studied in the framework of the Brueckner-Hartree-Fock theory including a three-body force. The energy per nucleon $E_{A}(\delta)$ calculated in the full range of spin polarization $\delta=\frac{\rho_{\uparrow}-\rho_{\downarrow}}{\rho}$ for symmetric nuclear matter and pure neutron matter fulfills a parabolic law. In both cases the spin-symmetry energy is calculated as a function of the baryonic density along with the related quantities such as the magnetic susceptibility and the Landau parameter $G_{0}$. The main effect of the three-body force is to strongly reduce the degenerate Fermi gas magnetic susceptibility even more than the value with only two body force. The EOS is monotonically increasing with the density for all spin-aligned configurations studied here so that no any signature is found for a spontaneous transition to a ferromagnetic state.
\end{abstract}




\section{INTRODUCTION}

Studies of spin-polarized nuclear and neutron matter have been mainly focussed on the possible onset of a ferromagnetic transition in the neutron star core. This transition could explain in fact the high intensity magnetic fields ( $10^{12}$ gauss) estimated from the timing observations in pulsars and magnetars (for a review see Ref. [四]). Besides this exciting issue the motivation for such studies can be based on a more general context of nuclear physics.

First of all, from the change of the energy per nucleon with the spin polarization one may extract a theoretical prediction for the spin symmetry energy, whose empirical value is so far quite uncertain. The nuclear matter instability against spin fluctuations is driven by the Landau parameter $G_{0}$ which is determined from the spin-symmetry energy. The value of this parameter is still a largely controversial topic and no agreement exists among the different approaches to the theory of nuclear matter [2]. Experimental information could come from spin giant resonances, which have not yet been clearly observed. Information could also come from heavy-ion collisions as soon as polarized heavy targets become available.

The second issue related to the study of spin-aligned states of nuclear matter is the propagation of neutrinos in neutron stars. It has been shown that the neutrino mean free path is strongly affected by the magnetic susceptibility. The latter is sizeably suppressed by the strong correlations in nuclear matter and, as a consequence, the mean free path might change sizeably and, eventually drop to zero in the presence of a ferromagnetic transition [3, 四].

There is a guess that the ferromagnetic transition could be a relativistic effect due to $\pi$-exchange and in fact all calculations, based on the relativistic mean field approch, predict this transition to occur in dense matter [5:6]. On the other hand, non-relativistic approaches [7] 10] do not support such a transition except Hartree-Fock calculations with phenomenological Skyrme-like forces (for a review see Ref. [4). This aspect cannot be disconnected from the problem of the in-medium nucleon-nucleon (NN) force, which is poorly known in dense matter due to the lacking of empirical constraints far above the saturation density. However important relativistic effects can be incorporated into the effective interaction via the three-body force associated with a virtual nucleon-antinucleon excitation [11]. Moreover non-relativistic calculations including only two-body forces miss the empirical saturation point of nuclear matter [12]. So it seems worthwhile to investigate the spin-aligned states of nuclear and neutron matter in the non-relativistic Brueckner theory with three-body forces. They contain not only the above mentioned relativistic contributions

but also nucleonic excitations which decisively enhance the agreement between theoretical and empirical saturation density [13. 


\section{FORMALISM}

The spin and isospin asymmetric nuclear matter (ANM) consists of spin-up neutrons $(n \uparrow)$, spin-down neutrons $(n \downarrow)$, spin-up protons $(p \uparrow)$ and spin-down protons $(p \downarrow)$ in different density states: $\rho_{n \uparrow}, \rho_{n \downarrow}, \rho_{p \uparrow}$, and $\rho_{p \downarrow}$, respectively. Therefore four parameters are required to specify a given configuration of spin and isospin ANM. The Fermi momenta of the four components are generally different from each other, and related to their respective densities by the following relation:

$$
\rho_{\lambda}=\frac{1}{6 \pi^{2}}\left(k_{F}^{\lambda}\right)^{3}
$$

where $\lambda$ denotes the $z$-components of isospin and spin, i.e., $\lambda=\left(\tau_{z}, \sigma_{z}\right)$. Instead of $\rho_{\lambda}$, one can use the following four parameters to identify a given spin and isospin state,

$$
\beta=\frac{\rho_{n}-\rho_{p}}{\rho}, \quad \delta_{n}=\frac{\rho_{n \uparrow}-\rho_{n \downarrow}}{\rho_{n}}, \quad \delta_{p}=\frac{\rho_{p \uparrow}-\rho_{p \downarrow}}{\rho_{p}},
$$

where $\rho, \rho_{n}$, and $\rho_{p}$ are total density, neutron density and proton density, respectively. The ratio $\beta$ is the isospin asymmetry parameter and $\delta_{n}$ and $\delta_{p}$ are the spin asymmetry parameters for neutrons and protons, respectively.

The starting point of the Brueckner-Bethe-Goldstone (BBG) approach is the reaction $G$-matrix. The $G$-matrix incoporates strong short-range correlations in nuclear medium by means of the infinite ladder diagram summation of the bare NN interaction. It satisfies the Bethe-Goldstone equation. The latter can be expressed for the spin-isospin ANM in the total angular-momentum basis as follows,

$$
\begin{aligned}
G_{L L^{\prime}}^{T S J, \lambda \lambda^{\prime}}\left(\omega, P ; q, q^{\prime} ; \rho, \beta, \delta_{n}, \delta_{p}\right) & =v_{L L^{\prime}}^{T S J}\left(q, q^{\prime}\right)+\frac{2}{\pi} \sum_{L^{\prime \prime}} \int q^{\prime \prime 2} \mathrm{~d} q^{\prime \prime} v_{L L^{\prime \prime}}^{T S J}\left(q, q^{\prime \prime}\right) \\
& \times \frac{\left\langle Q^{\lambda \lambda^{\prime}}\left(q^{\prime \prime}, P\right)\right\rangle}{\omega-e_{12}^{\lambda \lambda^{\prime}}\left(q^{\prime \prime}, P\right)+i \eta} G_{L^{\prime \prime} L^{\prime}}^{T S J, \lambda, \lambda^{\prime}}\left(\omega, P ; q^{\prime \prime}, q^{\prime} ; \rho, \beta, \delta_{n}, \delta_{p}\right)
\end{aligned}
$$

where $v_{L L^{\prime}}^{T S J}\left(q, q^{\prime}\right)$ are the partial wave components of the NN interaction, $\vec{P}=\vec{k}_{1}+\vec{k}_{2}=\vec{k}_{1}^{\prime}+\vec{k}_{2}^{\prime}$ is the total momentum, $\vec{q}=\frac{\vec{k}_{1}-\vec{k}_{2}}{2}$ and $\vec{q}^{\prime}=\frac{\vec{k}_{1}^{\prime}-\vec{k}_{2}^{\prime}}{2}$ the relative momenta of the two particles in their initial state and final state, respectively. The Pauli operator $Q^{\lambda \lambda^{\prime}}\left(\vec{q}^{\prime \prime}, \vec{P}\right)$ and the energy denominator $e_{12}^{\lambda \lambda^{\prime}}\left(\vec{q}^{\prime \prime}, \vec{P}\right)=\epsilon^{\lambda}\left(k_{1}^{\prime \prime}\right)+\epsilon^{\lambda^{\prime}}\left(k_{2}^{\prime \prime}\right)$ have been angle averaged in order to remove the coupling between different channels $\alpha=\{J S T\}$. It is worth noticing that the different components of the $G$-matrix differ in general from each other due to the dependence of the Pauli operator and energy denominator on the spin-isospin configuration $\left(\lambda, \lambda^{\prime}\right)$. The single particle energy is given by $\epsilon^{\lambda}(k)=\hbar^{2} k^{2} / 2 m+U^{\lambda}(k)$. The continuous choice for the 
auxilary potential $U^{\lambda}(k)$ is adopted in the present calculations since, on the one hand, it has been shown to provide a much faster convergence of the hole-line expansion than the gap choice [15], on the other hand, it decribes physically the single-particle potential felt by a nucleon in nuclear medium. In the continuous choice, $U^{\lambda}(k)$ is the real part of the on-shell mass operator, i.e.,

$$
U^{\lambda}(k)=R e \sum_{\vec{k}^{\prime}, \lambda^{\prime}} n^{\lambda^{\prime}}\left(k^{\prime}\right)\left\langle\vec{k} \lambda, \vec{k}^{\prime} \lambda^{\prime}\left|G\left[\omega=\epsilon^{\lambda}(k)+\epsilon^{\lambda^{\prime}}\left(k^{\prime}\right), P\right]\right| \vec{k} \lambda, \overrightarrow{k^{\prime}} \lambda^{\prime}\right\rangle_{A},
$$

where the subscript $A$ denotes antisymmetrization. For spin and isospin ANM, it is convenient to split it into two contributions as

$$
U^{\lambda}=U^{\lambda \lambda}+U^{\lambda \lambda^{\prime}}, \quad\left(\lambda^{\prime} \neq \lambda\right) .
$$

Each individul contribution is calculated by casting it into the partial wave expansion,

$$
\begin{aligned}
U^{\sigma_{z} \tau_{z}, \sigma_{z}^{\prime} \tau_{z}^{\prime}}(k) & =\int_{0}^{k_{F}^{\sigma_{z}^{\prime} \tau_{z}^{\prime}}} \mathrm{d}^{3} k^{\prime} \sum_{T S J} \sum_{L L^{\prime}} \sum_{S_{z} T_{z}} i^{L-L^{\prime}}\left[C\left(\frac{1}{2} \sigma_{z} \frac{1}{2} \sigma_{z}^{\prime} \mid S S_{z}\right)\right]^{2}\left[C\left(\frac{1}{2} \tau_{z} \frac{1}{2} \tau_{z}^{\prime} \mid T T_{z}\right)\right]^{2} \\
& \times \sum_{M_{L}} C\left(L^{\prime} M_{L} S S_{z} \mid J M_{L}+S_{z}\right) C\left(L M_{L} S S_{z} \mid J M_{L}+S_{z}\right) Y_{L^{\prime} M_{L}}^{*}(\hat{q}) Y_{L M_{L}}(\hat{q}) \\
& \times 2 G_{L L^{\prime}}^{T S J, \lambda \lambda^{\prime}}\left(\omega, P, q, q ; \rho, \beta, \delta_{n}, \delta_{p}\right) .
\end{aligned}
$$

The summation over partial wave states is physically constrained by the selection rule $S+$ $T+L=$ odd due to the Pauli principle and consequently the antisymmetrization of the $G$ matrix simply implies multiplication by a factor of 2 for the allowed partial wave channels. For spin symmetric case $\left(\delta_{n}=\delta_{p}=0\right.$ ), a spin-up neutron (proton) has the same Fermi momentum as a spin-down neutron (proton) and thus the single-particle potential felt by a nucleon does not depend on the direction of its spin. The summation on the spins of the two particles in the final state and the average of that in the initial state remove the non-diagonal contributions in angular-momentum from the single-particle potential. One easily finds

$$
\begin{aligned}
U^{\tau_{z} \tau_{z}^{\prime}}(k) & =\frac{1}{2} \sum_{\sigma_{z} \sigma_{z}^{\prime}} U^{\sigma_{z} \tau_{z}, \sigma_{z}^{\prime} \tau_{z}^{\prime}}(k) \\
& =\frac{1}{2} \int_{0}^{k_{F}^{\tau_{z}}} \mathrm{~d}^{3} k^{\prime} \sum_{T S J L} \sum_{T_{z}}\left[C\left(\frac{1}{2} \tau_{z} \frac{1}{2} \tau_{z}^{\prime} \mid T T_{z}\right)\right]^{2} \frac{2 J+1}{4 \pi} 2 G_{L L}^{T S J, \tau_{z} \tau_{z}^{\prime}}(\omega, P, q, q ; \rho, \beta) .
\end{aligned}
$$

For spin-asymmetric but isospin-symmetric nuclear matter, we have $\beta=0$ and $\delta_{n}=\delta_{p}=$ $\frac{\rho_{\uparrow}-\rho_{\downarrow}}{\rho}=\delta$. In this case, the single particle potential becomes,

$$
\begin{aligned}
U^{\sigma_{z}, \sigma_{z}^{\prime}} & =\int_{0}^{k_{F}^{\sigma_{z}^{\prime}}} \mathrm{d}^{3} k^{\prime} \sum_{T S J} \frac{2 T+1}{2} \sum_{L L^{\prime}} \sum_{S_{z}} i^{L-L^{\prime}}\left[C\left(\frac{1}{2} \sigma_{z} \frac{1}{2} \sigma_{z}^{\prime} \mid S S_{z}\right)\right]^{2} 2 G_{L L^{\prime}}^{T S J, \sigma_{z} \sigma_{z}^{\prime}}(\omega, q, q ; \rho, \delta) \\
& \times \sum_{M_{L}} C\left(L^{\prime} M_{L} S S_{z} \mid J M_{L}+S_{z}\right) C\left(L M_{L} S S_{z} \mid J M_{L}+S_{z}\right) Y_{L^{\prime} M_{L}}^{*}(\hat{q}) Y_{L M_{L}}(\hat{q}) .
\end{aligned}
$$


The present calculations will mainly consider this spin-polarized nuclear matter as well as the spin polarized neutron matter.

\section{RESULTS AND CONCLUSIONS}

We performed some calculations within the BHF self-consistent approach above described. The Argonne $V_{18}$ force is adopted as bare two-body interaction. This has been implemented by a microscopic three-body force, which is described in detail in Ref. 16] together with the average procedure to transform it into an effective two body force. In Fig. 1 the energy shift per nucleon $E_{A}(\delta, \rho)-E_{A}(0, \rho)$ in symmetric nuclear matter is reported as a function of the square of spin polarization $\delta^{2}$ for a set of densities. Due to the linear dependence on $\delta^{2}$, also reported in Refs. [9,10] one can write:

$$
E_{A}(\delta, \rho)=E_{A}(0, \rho)+\mathcal{E}_{\text {sym }}(\rho) \delta^{2}
$$

i.e. the spin dependence of the energy per nucleon can be simply expressed in terms of spin-symmetry energy

$$
\mathcal{E}_{\text {sym }}(\rho)=\frac{1}{2} \frac{\partial^{2} E_{A}(\delta, \rho)}{\partial \delta^{2}}
$$

in the density range here considered.

The $\delta^{2}$-law is mainly due to the BHF approximation (two-hole line terms only). The same behavior is in fact exhibited by the energy vs. isospin either [14]. The effects of three-hole line terms are rather small when adopting the continuous choice for the auxiliary potential [15]; this choice is also adopted for the present calculations. The slope of the energy shift is monotonically increasing with density so that no signature for a ferromagnetic phase transition in symmetric nuclear matter is expected. The effect of three-body force is to enhance this slope for densities above the saturation point. This effect is more clearly shown in the plot of $\mathcal{E}_{\text {sym }}$ vs. density in Fig. 1.

The magnetic susceptibility has been also calculated from the spin-symmetry energy

$$
\chi=\frac{\bar{\mu}^{2} \rho}{2 \mathcal{E}_{\text {sym }}},
$$

where $\bar{\mu}$ is the average of neutron and proton magnetic moments (in neutron matter $\bar{\mu}$ is the exact neutron magnetic moment). Usually one calculates the ratio of $\chi$ to $\chi_{F}, \chi_{F}$ being the magnetic susceptibility for a degenerate free Fermi gas. The effect of strong correlations in nuclear matter due to the two-body force is a reduction of $\chi$ with respect to $\chi_{F}$. This reduction increases with density up to a factor of 0.3 at $\rho=0.8 \mathrm{fm}^{-3}$. The above result is common to most Bruckner calculations [8, 11, 9, 10]. More pronounced is the queching due to three-body force. 
Fig. 2 also shows the Landau parameter $G_{0}$ describing the spin density fluctuations in the effective interaction. $G_{0}$ is simply related to the spin-symmetry energy or, equally, to the magnetic susceptibility by the relation

$$
\frac{\chi}{\chi_{F}}=\frac{m^{*}}{1+G_{0}}
$$

where $m^{*}$ is the effective mass. A magnetic instability would require $G_{0}<-1$ which is analogous to the condition $F_{0}<-1$ for the mechanical instability giving rise to the liquidvapour phase transition. But, the value of $G_{0}$ vs. density from the BHF calculations is always positive and monotonically increasing up to the highest density. The three-body force pushes up the curve of $\chi$. This result is in strong disagreement with the prediction with Skyrme forces. This is not a complete surprise since Skyrme forces are only well suited in the proximity of the empirical saturation point. Astonishing is the strong disagreement on this respect with the relativistic approaches because the three-body forces contain already important relativistic effects [16]. The accurate knowledge of $G_{0}$ should lead to reliable predictions on the spin and spin-isospin giant modes as well as spin-spin part of the optical potential [8].

The above calculations have been also repeated for the case of pure neutron and reported in Fig. 2. The same conclusions can be drawn as to the absence of the ferromagnetic phase transition and the quenching of the magnetic susceptibility caused by the strong correlations from the two- and three-body forces. This quenching should have a strong influence on the neutrino propagation in dense matter such as supernovae and neutron stars. In the case of the transition to a ferromagnetic state it has been shown that the mean free path could drop to zero [3] that could have remarkable consequences as, for instance, on the neutron star cooling. 


\section{REFERENCES}

[1] A. Reisenegger, Magnetic Fields across the Hertzsprung-Russell Diagram, arXiv: astroph/0103010.

[2] U. Lombardo, in "Nuclear Methods and the Nuclear Equation of State", Ed. M. Baldo, World Scientific, Singapore, 1999, p.458 and references therein quoted.

[3] J. Margueron, J. Navarro, N. van Giai and W. Jiang, Neutrino propagation in neutron matter and the nuclear equation of state, to appear in "The Nuclear Many-body Problem 2001", NATO Science Series II (Kluwer Academic Publishers).

[4] J. Margueron, PhD thesis (2001), Paris.

[5] P. Bernardos, S. Marcos, R. Niembro and M. L. Quelle, Phys. Lett. B356 (1995) 175.

[6] Tomoyuki Maruyama and Toshitaka Tatsumi, Nucl. Phys. A693 (2001) 710.

[7] P. Haensel, Phys. Rev. C11 (1975) 1822.

[8] J. Cugnon, P. Deneye and A. Lejeune, Nucl. Phys. A485 (1988) 271.

[9] I. Vidana, A. Polls and A. Ramos, Phys. Rev. C65 (2002) 035804.

[10] I. Vidana and I. Bombaci, Equation of state and magnetic susceptibility of spin polarized isospin asymmetric nuclear matter, arXiv: nucl-th/0203061.

[11] A. Lejeune, U. Lombardo and W. Zuo, Phys. Lett. 477 (2000) 45.

[12] M. Baldo, in "Nuclear Methods and the Nuclear Equation of State", Ed. M. Baldo, World Scientific, Singapore, 1999, p. 1 and references therein quoted.

[13] W. Zuo, A. Lejeune, U. Lombardo and J. F. Mathiot, arXiv: nucl-th/0202077, to appear in Nucl. Phys. A.

[14] W. Zuo, I. Bombaci and U. Lombardo, Phys Rev. C60 (1999) 024605.

[15] H. Q. Song, M. Baldo, G. Giansiracusa and U. Lombardo, Phys. Rev. Lett. 81 (1998) 1584 .

[16] P. Grangé, A. Lejeune, M. Martzolff, and J. -F. Mathiot, Phys. Rev. C40 (1989) 1040. 


\section{FIGURES}

FIG. 1. Uper windows: The EOS of spin-asymmetric nuclear matter as a function of spin-asymmetry at five values of density, predicted by Brueckner Hartree-Fock calculations adopting pure $\mathrm{AV}_{18}$ two-body force (right-uper window) and $\mathrm{AV}_{18}$ plus the $\mathrm{TBF}$ (left-uper window). Right-Lower window: density dependence of spin-symmetry energy for both cases with the TBF (solid curve) and without the TBF(dash curve). Left-lower window: Magnetic suscepbility $\chi / \chi_{0}$ (curves with symbol) and Landau parameter $G_{0}$ (curves without symbol) as functions of density.

FIG. 2. The same as Fig.1 for spin-polarized neutron matter. 
Spin $\mathrm{E}_{\mathrm{sym}}(\rho)(\mathrm{MeV})$

$\mathrm{E}_{\mathrm{A}}(\delta, \rho)-\mathrm{E}_{\mathrm{A}}(0, \rho)(\mathrm{MeV})$
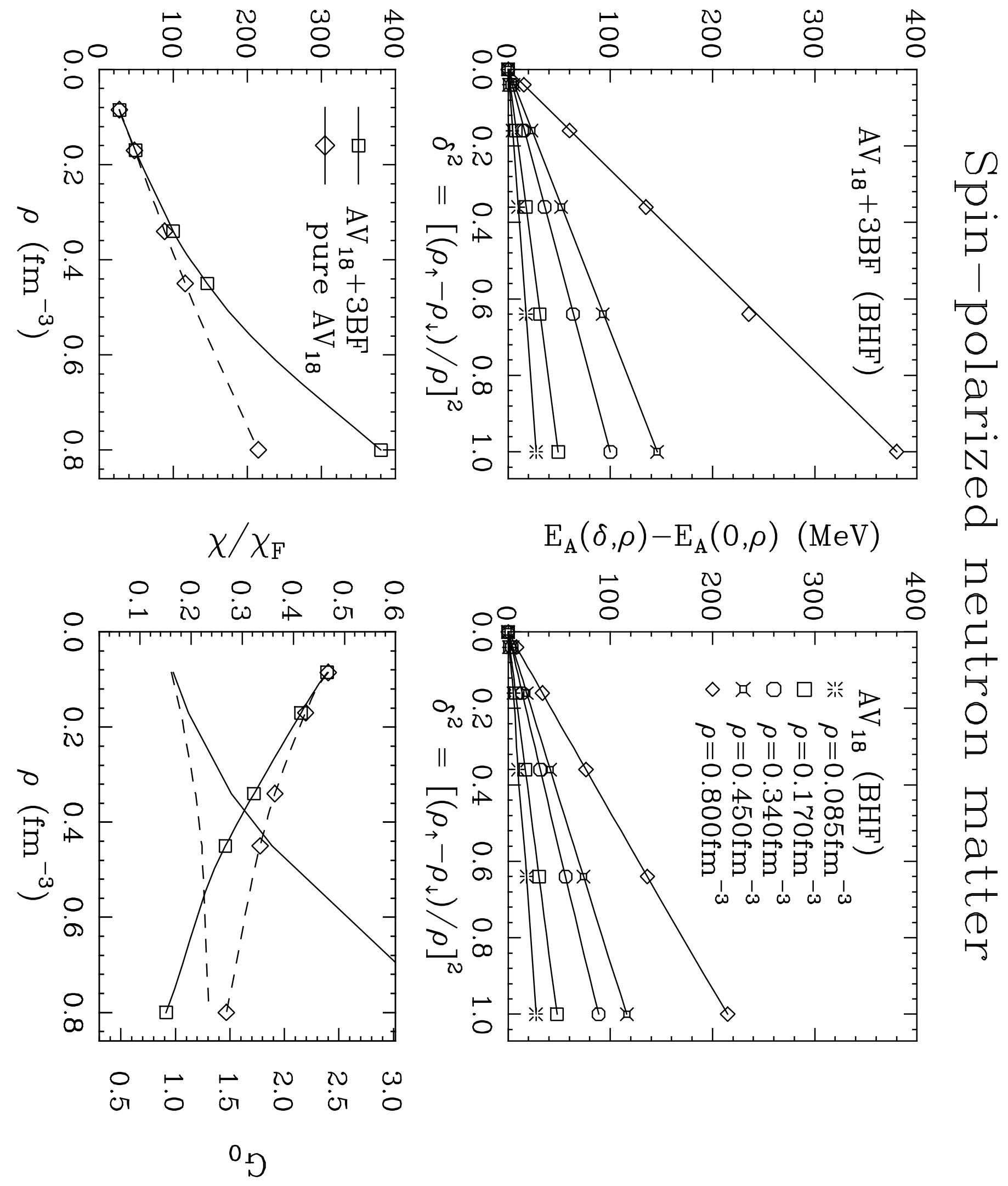
Spin $\mathrm{E}_{\mathrm{sym}}(\rho)(\mathrm{MeV})$

$\mathrm{E}_{\mathrm{A}}(\delta, \rho)-\mathrm{E}_{\mathrm{A}}(0, \rho)(\mathrm{MeV})$
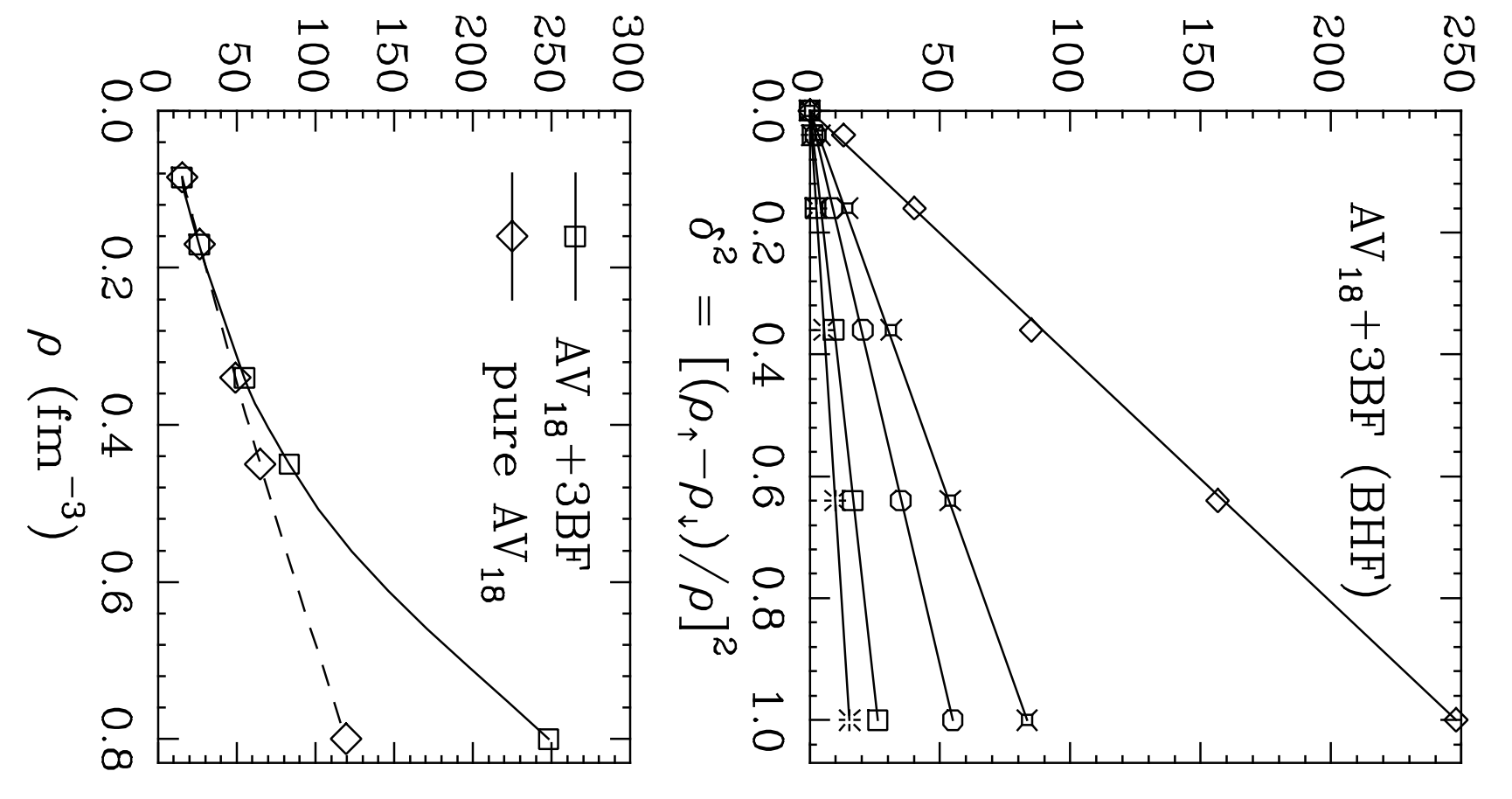

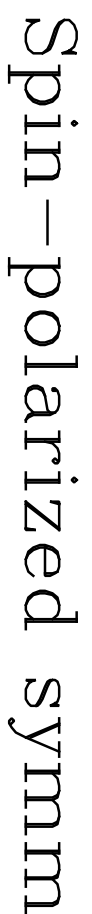
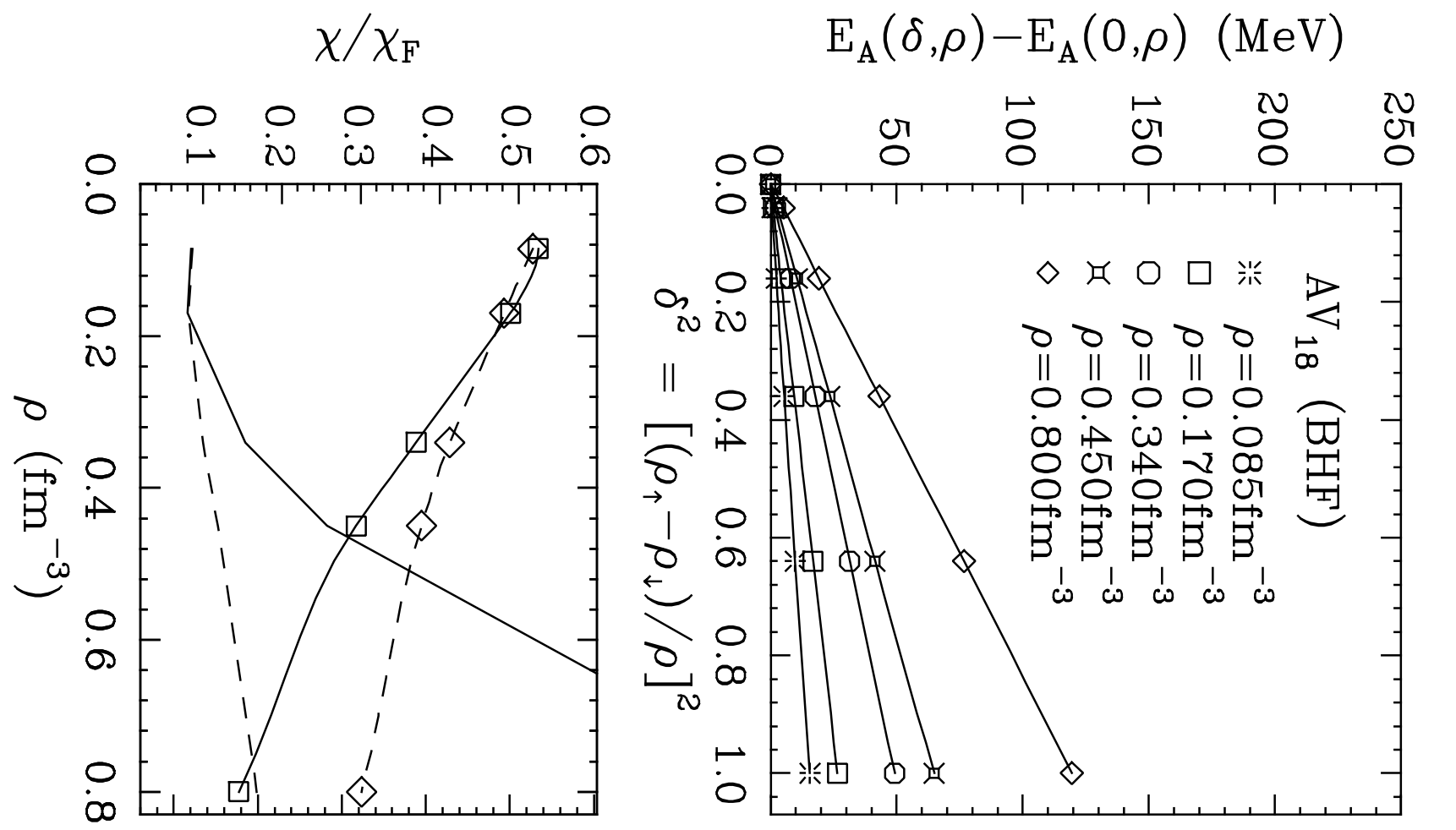

$\stackrel{2}{\frac{1}{3}}$

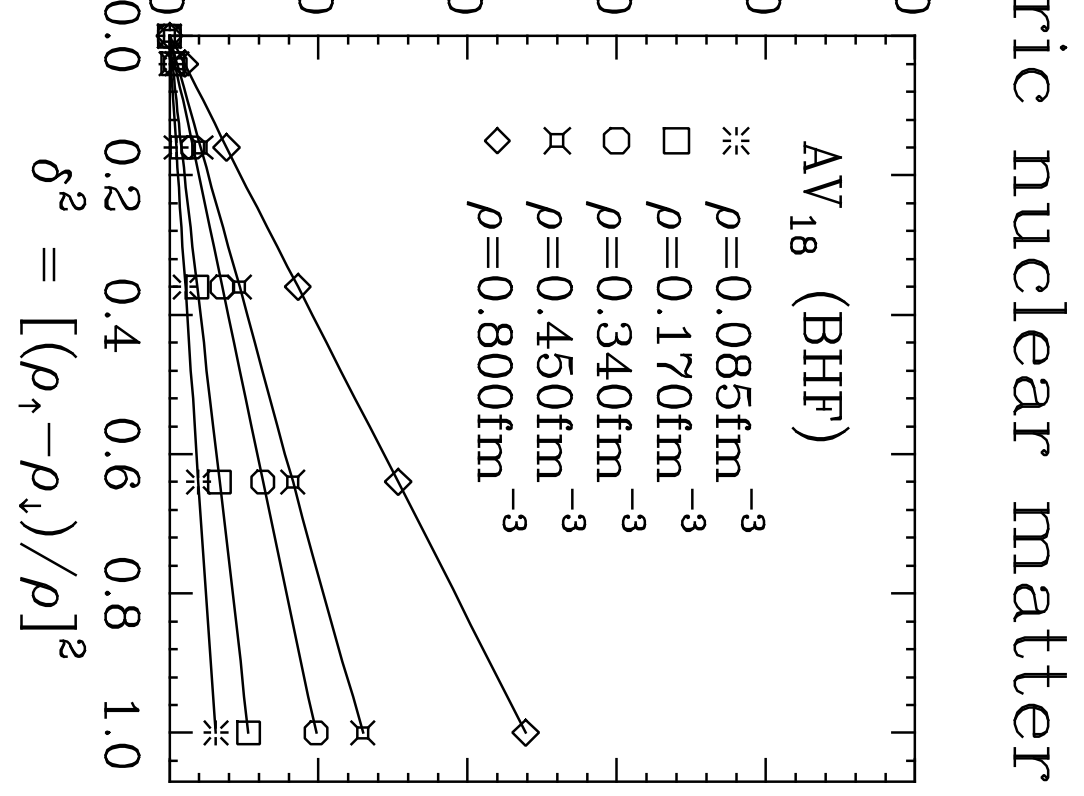

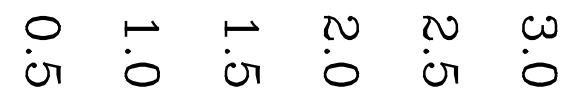

